\title{
Analyse fiabiliste de systèmes mécaniques par arbres de défaillance physiques et probabilistes
}

\author{
Anthony Hähnel ${ }^{1,2, a}$, François Rieuneau ${ }^{1}$ et Maurice Lemaire ${ }^{2}$ \\ 1 Renault s.a.s, 67 rue des bons raisins, 92508 Rueil Malmaison, France \\ 2 LaMI UBP \& IFMA, Campus de Clermont-Ferrand « Les Cézeaux », BP 265, 63175 Aubière Cedex, France
}

Reçu le 20 mars 2004, accepté le 5 janvier 2005

\begin{abstract}
Résumé - Les méthodes mécano-fiabilistes ont démontré leur efficacité dans la prise en compte des incertitudes lors de la conception de systèmes mécaniques. Elles reposent sur une modélisation mécanique du comportement du système vis-à-vis des modes de défaillance et sur une description probabiliste des paramètres incertains intervenant dans les modèles de comportement. La perte de la fonction que doit satisfaire un système mécanique résulte de l'occurrence de situations potentielles telles que celles mises en évidence au cours d'une analyse par arbre de défaillance (Perfect Fault Tree Analysis - P-FTA), par exemple. L'intérêt d'un tel arbre est d'identifier chaque scénario de défaillance au niveau le plus élémentaire possible. À cette démarche qualitative, nous associons une démarche quantitative basée sur les méthodes probabilistes de fiabilité afin de mesurer l'importance de chaque variable et de chaque combinaison de variables dans la perte de fonction. Nous proposons donc une méthode d'analyse fiabiliste de systèmes mécaniques au moyen d'arbres de défaillance physiques et probabilistes. La méthodologie proposée est illustrée au moyen d'une application concernant la rupture d'un arbre de boîte de vitesses.
\end{abstract}

Mots clés : Arbre de défaillance / couplage mécano-fiabiliste / analyse de fiabilité / analyse d'importance et de sensibilité / fonction de densité de probabilité / méthode FORM / simulation de Monte Carlo / arbre de boîte de vitesses

\begin{abstract}
Mechanical-based reliability analysis using fault trees. Structural reliability methods have proven their convenience to take into account all type of uncertainties during the design step of a mechanical system. They are based on a mechanical modelization of the physical behaviour of a system according to considered failure modes. They are also based on a probabilistic representation of input parameters introduced in the model. The lost of functionality that a mechanical system must fulfill follows the occurrence of potential situations that can be identified at the most elementary stage using well-known safety assessment tools like Perfect Fault Trees Analysis (P-FTA). We introduce an extended quantitative approach based on probabilistic reliability methods and transfer block treatment. The aim is to determinate the weight of each random variable or combination of variables involved in the loss of functionnality and to monitor the propagation of uncertainties. In other words, we proposed a framework for mechanical-based reliability analysis using fault trees. An application to the fracture behaviour of a transmission idler shaft is used to illustrate the whole process.
\end{abstract}

Key words: Fault tree analysis / mechanical modeling / reliability analysis / importance and sensitivity analysis / probability density function / FORM / Monte Carlo simulation / transmission idler shaft

\section{Introduction}

Un système mécanique est dit défaillant lorsqu'il ne peut plus assurer la fonction pour laquelle il a été conçu. Les études de sûreté de fonctionnement [1-3] ont pour but l'identification des situations potentielles entraînant

a Auteur correspondant : anthony.hahnel@renault.com la défaillance d'un système ainsi que la caractérisation de leur occurrence, ceci avec un objectif de sûreté mais aussi avec le souci de contrôler et de maîtriser la durée de vie. Renault s.a.s modélise des problèmes de fiabilité mécanique au moyen d'arbres de défaillance physiques (Perfect Fault Tree Analysis - P-FTA). Ils ont le mérite de proposer une description exhaustive des mécanismes physiques critiques et de leurs combinaisons susceptibles 


\begin{tabular}{|c|c|}
\hline \multicolumn{2}{|c|}{ Nomenclature } \\
\hline \multicolumn{2}{|c|}{ Symboles latins } \\
\hline$a_{i}$ & coefficients polynomiaux \\
\hline$b_{1}, b_{2}$ & bornes d'intégration \\
\hline$c, c^{(n)}$ & constante de normalisation, d'ordre $n$ \\
\hline$c_{X}^{(n)}$ & $\begin{array}{l}\text { constante de normalisation d'ordre } n \\
\text { de la variable aléatoire } X\end{array}$ \\
\hline$G_{k}^{(l)}$ & $k^{\text {ième }}$ variable de performance de niveau $l$ \\
\hline$\left[J_{\{u\},\{x\}}^{n}\right]$ & $\begin{array}{l}\text { Jacobien de la transformation } \\
\{x\} \longmapsto\{u\}=u(\{x\})\end{array}$ \\
\hline & moyenne de la variable aléatoire $X$ \\
\hline$M_{k}^{(l)}$ & $k^{\text {ième }}$ modèle mathématique de niveau $l$ \\
\hline$n v a_{l}$ & nombre de variables au niveau $l$ \\
\hline & nombre de nouvelles variables au niveau $l$ \\
\hline & probabilité de défaillance \\
\hline$S_{k}^{(1)}$ & $k^{\text {ième }}$ variable de type seuil de niveau $l$ \\
\hline$\left\{X^{(l)}\right\}$ & vecteur des paramètres au niveau $l$ \\
\hline \multicolumn{2}{|c|}{ Symboles grecs } \\
\hline$\{\alpha\}$ & vecteur de cosinus directeurs \\
\hline$\beta$ & indice de fiabilité \\
\hline $\begin{array}{l}\{\gamma\} \\
\delta(x)\end{array}$ & $\begin{array}{l}\text { vecteur d'importance des variables physiques } \\
\text { erreur d'approximation }\end{array}$ \\
\hline$\lambda$ & seuil d'étude \\
\hline$\sigma_{X}$ & écart-type de la variable aléatoire $X$ \\
\hline
\end{tabular}

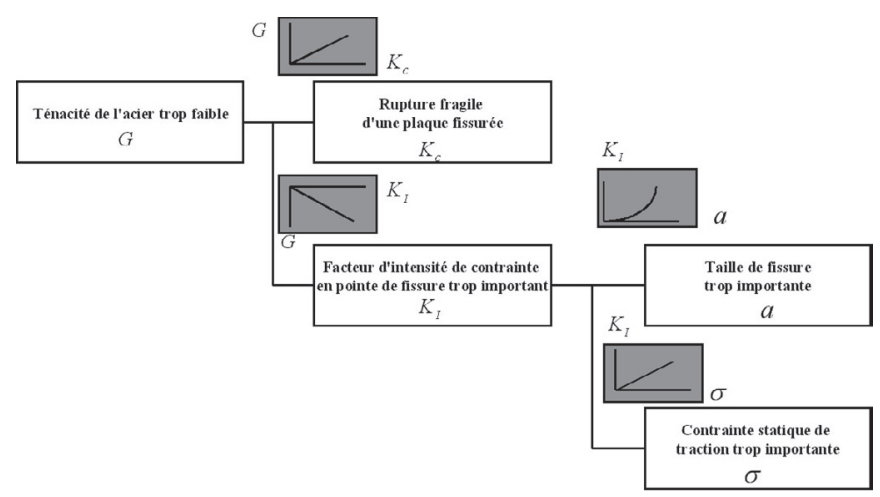

Fig. 1. Un exemple d'arbre de défaillance « réduit» de type PFTA.

de favoriser la défaillance d'un système complexe. Les relations (au premier ou second ordre) entre mécanismes ou paramètres successifs, sont approchées par des graphes de tendance tels que le montre la figure 1. Sa lecture s'effectue de droite à gauche. Une insuffisance de ténacité résulte d'une concentration de contrainte ou d'une fragilité; le facteur d'intensité de contrainte résulte de la contrainte statique et de la taille de fissure. Des graphes qualitatifs indiquent le sens des évolutions en chaque nœud.

Les limites de cette démarche résident essentiellement dans son caractère purement descriptif et qualitatif. On propose ici de lui donner une dimension quantitative par la mise en œuvre d'une approche mécanique et stochastique.
La question est alors de savoir comment exploiter une telle modélisation par P-FTA pour prendre en compte l'effet des incertitudes de caractérisation et des dispersions des paramètres de conception sur l'occurrence d'une défaillance potentielle. Les méthodes communément utilisées, pour prendre en compte les variabilités inhérentes aux paramètres de conception et aux phénomènes physiques lors de la simulation numérique d'un système mécanique, reposent sur des outils tels que les techniques de Monte Carlo, les méthodes de moments ou encore les méthodes FORM/SORM [4].

La méthode que nous proposons s'inspire des outils de calcul exploitées dans le cadre des méthodes mécano-fiabilistes [5]. L'objectif est d'estimer la probabilité d'occurrence d'un événement redouté, renseigner à un niveau quelconque de l'arbre et d'identifier l'influence de n'importe quelle variable amont tout en conservant une modélisation assez fine de la physique des mécanismes aboutissant à la défaillance. Chaque variable, et donc chaque phénomène qu'elle représente, est ainsi caractérisée par sa fonction de densité de probabilité et par une analyse de sensibilité par rapport aux paramètres qui l'ont engendrée. Ceci permet de conclure quant à l'importance relative des divers phénomènes impliqués dans un scénario de défaillance.

\section{Construction d'un P-FTA mécano-fiabiliste}

L'information descriptive que fournit une analyse par arbre de défaillance physique et la connaissance qualitative des phénomènes physiques et des grandeurs physiques mises en jeu sont utilisées afin d'obtenir une représentation qui va permettre son traitement quantitatif probabiliste. Autrement dit, on construit un système de blocs de transfert (Fig. 2). Chaque bloc de transfert correspond à un modèle mécanique précis dans lequel on distingue clairement les variables d'entrée et de sortie du modèle. L'intérêt d'une telle représentation est bien entendu de faciliter la mise en place d'une méthode d'exploitation quantitative et stochastique d'arbres de défaillance. La méthode que nous proposons ci-dessous repose sur l'approche mécano-fiabiliste d'un scénario de défaillance et sur la méthode d'approximation de fonctions de densité de variables aléatoires par exponentielle d'un polynôme (EPM) [6].

\subsection{Principe de décomposition de l'arbre}

\subsubsection{Niveau élémentaire (0)}

Au niveau le plus élémentaire se situent les variables aléatoires de base du problème. Ce sont les variables pour lesquelles le concepteur estime disposer d'une connaissance statistique suffisante et pour lesquelles, il ne juge pas nécessaire de rechercher une information supplémentaire fonction de leur origine physique. Ainsi, la résistance à la rupture d'un composant mécanique, par exemple, peut être considérée comme une variable de base 


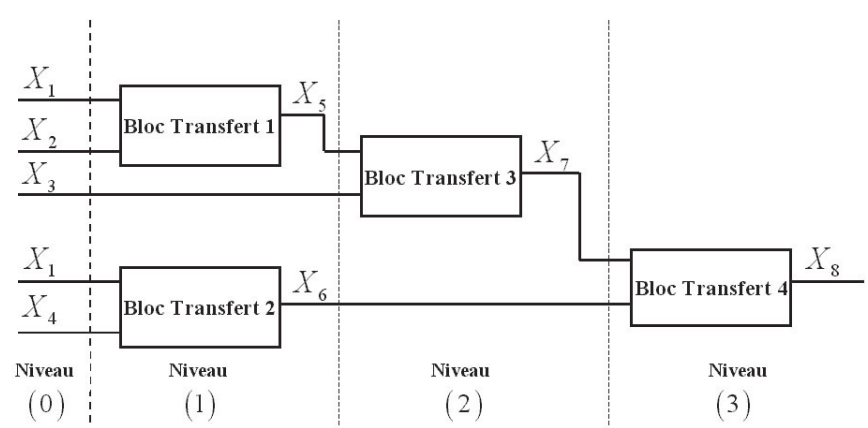

Fig. 2. Exemple de représentation par blocs de transfert d'un arbre de défaillance simple.

mais elle pourrait également être considérée comme la variable résultant de la combinaison des divers constituants de sa matière.

On note $\left\{X^{(0)}\right\}$ le vecteur de ces variables en nombre $n v a_{0}$. En toute rigueur, il serait nécessaire de connaître la densité conjointe de probabilité de ces variables, ce qui est irréaliste. Dans la pratique, au mieux, seront disponibles des informations permettant d'identifier les lois de probabilité marginales et les coefficients de corrélation de ces variables aléatoires. La quantité d'information ne permet bien souvent que des estimations des paramètres des lois marginales qui pourraient également être considérés comme des variables aléatoires et être ajoutés dans le vecteur $\left\{X^{(0)}\right\}$ selon les méthodes proposées par Pendola [7]. Cependant, ceci est de nature à complexifier le problème. L'hypothèse de travail retenue à ce stade consiste à disposer des lois marginales à paramètres déterminés et éventuellement des corrélations.

\subsubsection{Niveau (1)}

Le niveau (1) est constitué des variables du niveau (0) auxquelles s'ajoutent les variables engendrées par le passage du niveau (0) au niveau (1). Notons $N c_{1}$ le nombre de variables $S_{k}, k=1, \ldots, N c_{1}$ ainsi créées. Chaque variable $S_{k}$ résulte d'une transformation mécanique, qui n'est autre que le modèle mécanique associé au bloc de transfert correspondant

$$
\left\{X^{(0)}\right\} \text { modèle mécanique } k^{(1)} S_{k}^{(1)}=M_{k}^{(1)}\left(\left\{X^{(0)}\right\}\right)
$$

où le $k^{\text {ème }}$ modèle mécanique représentant une transformation de l'état (0) à l'état (1) est noté $M_{k}^{(1)}, k=$ $1, \ldots, N c_{1}$.

Les variables $\left\{X^{(1)}\right\}$ sont donc définies par

$$
\begin{aligned}
\left\{X^{(0)}\right\}_{n v a_{0} \text { variables }} & \stackrel{M_{k}^{(1)}}{\longrightarrow}\left\{X^{(1)}\right\}_{n v a_{0}+N c_{1} \text { variables }}= \\
& \left\{\begin{array}{l}
\left\{X^{(0)}\right\} \\
\left\{S^{(l)}\right\}
\end{array}\right\}, \text { avec } S_{k}^{(l)}, k=1, \ldots, N c_{1}
\end{aligned}
$$

La question est alors de déterminer les propriétés statistiques des variables $\left\{X^{(1)}\right\}$. La méthode utilisée dans cet article est présentée ci-après.

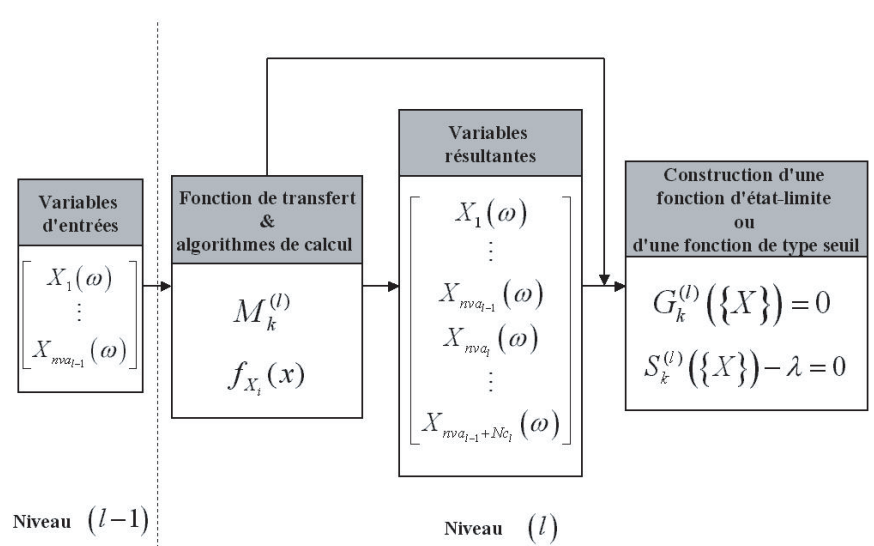

Fig. 3. Représentation du modèle mécanique du passage du nœud $(l)$ au nœud $(l+1)$.

\subsubsection{Niveau $(/)$}

D'une manière générale, la procédure conduit à l'obtention de variables $\left\{X^{(l)}\right\}$ en nombre $n v a_{l}=n v a_{0}+$ $\sum_{j=1}^{l} N c_{j}$. Ces variables $\left\{X^{(l)}\right\}$ peuvent être la cause de défaillances au niveau supérieur. Elles entrent alors dans un scénario de défaillance que l'on note $G_{k}^{(l+1)}\left(\left\{X^{(l)}\right\}\right)$. Les variables $G_{k}$ sont un type particulier de variables $S_{k}$ qui correspondent à des états-limites. On remarque qu'en considérant la différence $S_{k}-\lambda$ où $\lambda$ est un seuil d'étude donné on construit aussi un état-limite. La souplesse de cette représentation permet la détermination des sensibilités de n'importe quel type de variable $G_{k}$ ou $S_{k}$ que ce soit à la défaillance ou à un seuil donné.

\subsection{Procédure de passage d'un niveau de l'arbre}

Les étapes fondamentales nécessaires au passage d'un nœud de niveau $(l)$ peuvent être résumées selon le schéma suivant illustré en figure 3 :

1. tout d'abord, les $n v a_{l-1}$ variables aléatoires $\left\{X^{(l-1)}\right\}$ sont supposées connues par leur fonction de densité de probabilité; une procédure numérique est au moins apte à construire point par point leur densité.

2. Les transformations mécaniques $M_{k}^{(l)}, k=1, \ldots, N_{c_{1}}$ du noud $(l)$ associent $N_{c_{1}}$ nouvelles variables aux variables $\left\{X^{(l-1)}\right\}$ déjà identifiées. Ces nouvelles variables sont notées $S_{k}^{(l)}$ si elles correspondent à des quantités physiques ou $G_{k}^{(l)}$ si elles correspondent à une fonction de performance ou fonction d'état-limite. La construction du vecteur $\left\{X^{(l)}\right\}$ résulte donc de la procédure suivante

$$
\begin{aligned}
\left\{X^{(l-1)}\right\}_{n v a_{l-1} \text { variables }} \stackrel{M_{k}^{(l)}}{\longrightarrow}\left\{X^{(l)}\right\}_{n v a_{l}=n v a_{l-1}+N c_{l} \text { variables }} \\
=\left\{\begin{array}{c}
\left\{X^{(l-1)}\right\} \\
\left\{S^{(l)}\right\} \\
\left\{G^{(l)}\right\}
\end{array}\right\}
\end{aligned}
$$


3. Les variables $X_{i}^{(l)}, i=n v a_{l-1} \ldots n v a_{l}$ de type $S_{k}^{(l)}$ ou $G_{k}^{(l)}$ sont identifiées par le calcul de leurs moments à l'aide d'une quadrature

$$
\begin{aligned}
\mathbf{E}\left[X_{i}^{(l)}\right]= & \\
& \left.\int_{\mathbb{R}^{n_{l}-1}} M_{k}^{(l)}\left(\left\{X^{(l-1)}\right\}\right) f_{\left\{X^{(l-1)}\right.}\right\} \\
& \times\left(\left\{x^{(l-1)}\right\}\right) \mathrm{d} x_{1} \ldots \mathrm{d} x_{n v a_{l}-1}
\end{aligned}
$$

$$
\begin{aligned}
\mathbf{E}\left[\left(X_{i}^{(l)}-\mathbf{E}\left[X_{i}^{(l)}\right]\right)^{j}\right] \\
=\int_{\mathbb{R}^{n_{l}-1}}\left(M_{k}^{(l)}\left(\left\{X^{(l-1)}\right\}-\mathbf{E}\left[X_{i}^{(l)}\right]\right)\right)^{j} \\
\times f_{\left\{X^{(l-1)}\right\}}\left(\left\{x^{(l-1)}\right\}\right) \mathrm{d} x_{1} \ldots \mathrm{d} x_{n v a_{l}-1}
\end{aligned}
$$

d'où les paramètres caractéristiques (moyenne, coefficient de variation, asymétrie - skewness, aplatissement - kurtosis, ...) sont déduits.

4. La connaissance de ces moments va permettre la construction de la fonction de densité de probabilité.

Des analyses de fiabilité et de sensibilité peuvent alors être conduites en considérant des fonctions d'étatslimites de la forme $X_{i}^{(l)}-\lambda_{i}=0, i=n v a_{l-1} \ldots n v a_{l}$ et en utilisant les fonctions de densité approchées des variables générées au cours de l'arbre (bien entendu, $\lambda_{i}=0$ quand $X_{i}^{(l)}$ est une variable de type $\left.G_{k}^{(l)}\right)$. On remarque également que la probabilité de défaillance et l'indice de fiabilité associé à une fonction de performance quelconque peuvent être déterminés soit en utilisant directement les fonctions de densité approchées soit par la mise en œuvre d'analyses FORM/SORM classiques. L'impact de chacune des variables et son importance dans un scénario de défaillance donné sont ainsi clairement identifiés.

\subsection{Approximation des densités de probabilité par la méthode de l'exponentielle d'un polynôme (EPM)}

\subsubsection{L'EPM, une méthode candidate parmi d'autres}

L'approximation de fonctions de densité de probabilité de variables aléatoires quelconques reste un problème ouvert. Dans [8-10], on propose d'approcher la fonction de densité de probabilité d'une variable aléatoire au moyen de familles de fonctions paramétrées, le type de fonction ainsi que ses paramètres étant définis grâce aux moments de la variable aléatoire (famille de Johnson et famille de Pearson). Ces deux méthodes sont comparées dans [11] à celle des séries de Gram-Charlier qui suppose qu'une densité de probabilité peut être approximée par une série faisant intervenir les dérivées de la loi normale et les moments de la variable aléatoire. Dans [12], il est également suggéré d'utiliser des séries de fonctions; entre autres, la méthode des séries de Edgeworth faisant intervenir les cumulants de la variable aléatoire (coefficients issus du développement de Taylor de sa fonction caractéristique) ainsi que le produit de polynômes d'Hermite et de la loi normale centrée réduite est présentée. Dans [13], on raisonne sur une variable aléatoire centrée réduite $X_{0}$ et sur l'existence de la transformation $U(t)=g^{-1}\left(X_{0}(t)\right)$, où $U$ est une variable normale de moyenne nulle, d'écarttype unitaire et $g$ une fonction monotone. Connaissant les $N$-premiers moments centrés de $X_{0}, g$ peut être approximée par une suite de polynômes d'Hermite. Dans [14] et [15], en supposant que l'on dispose d'un échantillon de données, on propose d'approcher la fonction de densité de la variable aléatoire par une combinaison linéaire de B-splines. De récents développements $[16,17]$ ouvrent de nouvelles possibilités sur la base d'une décomposition par des polynômes d'Hermite.

Un trop-plein de méthodes signifie simplement qu'aucune ne s'est imposée aujourd'hui de manière définitive. À travers notre expérience propre et celle de Pendola [7] ou de Sudret et al. [18], c'est la méthode EPM décrite par Er dans [6] qui apparaît la plus efficace et la plus souple d'emploi. L'EPM consiste à approcher la fonction de densité $f_{X}(x)$ d'une variable aléatoire $X$ par une fonction de la forme $c e^{Q(x)}$ où $Q(x)$ est une fonction polynomiale et $c$, une constante de normalisation. Le challenge demeure la détermination des coefficients du polynôme $Q(x)$.

\subsubsection{Formulation}

Soit $p_{X}(x)$ la fonction de densité de probabilité de la variable aléatoire $X$. Cette densité $p_{X}(x)$ est supposée définie et continue sur $\mathbb{R} \cdot p_{X}(x)$ est approchée par $q(x)=$ $\hat{p}_{X}(x)$ également définie sur $\mathbb{R}$ par

$$
q(x)=c e^{Q(x)}
$$

où $c$ est une constante de normalisation donnée par

$$
c=\frac{1}{\int_{-\infty}^{+\infty} e^{Q(x)} \mathrm{d} x}
$$

et $Q(x)$ un polynôme tel que

$$
Q(x)=\left\{\begin{array}{cc}
\sum_{i=1}^{\infty} a_{i} x^{i} & x \in \mathbb{R} \\
-\infty & x \rightarrow \pm \infty
\end{array}\right.
$$

où $a_{i}, i=1,2, \ldots$ sont des paramètres inconnus à déterminer. L'erreur d'approximation commise, $\delta(x) \in$ $\mathbf{L}_{2}(\mathbb{R})$ (ensemble des fonctions de carré intégrable sur $\mathbb{R}$ ), est définie par

$$
\delta(x)=\left[p_{X}(x)-q(x)\right] \cdot e^{|x| \frac{1}{r}}
$$

où $r$ est un entier positif « grand ». Il est montré dans [6] que d'après la théorie de l'approximation [19], $\delta(x)=0$ 
(i.e. $\left.p_{X}(x)=q(x)\right)$ si les équations suivantes sont vérifiées

$$
\begin{aligned}
\sum_{i=1}^{\infty} i \mu_{i+j-1}^{\prime} a_{i} & =-j \mu_{j-1}^{\prime} \quad j=1,2 \ldots \\
\sum_{i=1}^{\infty} i \mu_{i-1}^{\prime} a_{i} & =0
\end{aligned}
$$

où $\mu_{k}^{\prime}$ représente le moment d'ordre $k$ de la variable $X$ défini par

$$
\mu_{k}^{\prime}=\mathbf{E}\left[X^{k}\right]=\int_{-\infty}^{+\infty} x^{k} p_{X}(x) \mathrm{d} x
$$

\subsubsection{Mise en œuvre}

Dans la pratique, il est nécessaire d'utiliser une forme tronquée de $q(x)$ définie de la manière suivante

$$
\begin{gathered}
q^{(n)}(x)=c^{(n)} e^{Q^{(n)}(x)} \text { avec } Q^{(n)}(x) \\
=\left\{\begin{array}{cc}
\sum_{i=1}^{n} a_{i} x^{i} & x \in\left[b_{1}, b_{2}\right] \\
-\infty & \text { sinon }
\end{array}(n \geq 2)\right. \\
c^{(n)}=\frac{1}{\int_{b_{1}}^{b_{2}} e^{Q^{(n)}(x)} \mathrm{d} x}
\end{gathered}
$$

où les paramètres inconnus $a_{i}$ sont maintenant au nombre fini de $n$. Les expressions tronquées des équations (1) et (2) sont utilisées pour leur détermination

$$
\begin{aligned}
\sum_{i=1}^{n} i \mu_{i+j-1}^{\prime} a_{i} & =-j \mu_{j-1}^{\prime} \quad j=1,2, \ldots, n-1 \\
\sum_{i=1}^{n} i \mu_{i-1}^{\prime} a_{i} & =0
\end{aligned}
$$

Ainsi, les coefficients $a_{i}$ sont solutions d'un système linéaire d'équations construit à partir des $n$ premiers moments de la variable aléatoire $X$. La constante $c$ de normalisation peut être facilement calculée une fois les valeurs de $b_{1}$ et $b_{2}$ déterminées. D'après [6], l'expérience montre que la contribution de la grandeur $e^{Q^{(n)}(x)}$ à la constante de normalisation $c^{(n)}$ définie dans l'équation (5) devient négligeable dès lors que la valeur de $X$ est supérieure à $m_{X}+4 \sigma_{X}$ ou inférieure à $m_{X}-4 \sigma_{X}$, où $m_{X}$ est la valeur moyenne de $X$ et $\sigma_{X}$ son écart-type. Dans [7], on suggère de fixer $b_{1}$ et $b_{2}$ respectivement à $m_{X}-5 \sigma_{X}$ et $m_{X}+5 \sigma_{X}$. On en conclut donc que le choix de $b_{1}$ et $b_{2}$ doit être ajusté et affiné au cas par cas pour obtenir la meilleure précision possible.

Connaissant la fonction de densité de probabilité d'une variable aléatoire $X$ à partir de cette méthode EPM, il est possible d'obtenir simplement une estimation point par point de sa fonction de distribution sur $\left[b_{1}, b_{2}\right]$ au moyen de la relation

$$
\hat{P}_{X}(x)=\int_{b_{1}}^{x} \hat{p}_{X}(t) \mathrm{d} t, \quad x \in\left[b_{1}, b_{2}\right]
$$

\subsubsection{Discussion sur l'EPM}

La méthode EPM est sensible à la précision et à l'ordre des moments utilisés pour l'approximation. Celle-ci est d'autant plus efficace que la précision sur les moments est bonne et que l'ordre $n$ est grand. Cependant, il est difficile d'estimer finement des moments d'ordre élevé. Il est également nécessaire de souligner que la résolution du système linéaire d'équations (6) se complexifie et est numériquement instable dès lors que le nombre de moments devient trop important. Dans la mise en œuvre de la présente méthodologie, on se limite à un développement à l'ordre $n=5$, ce qui implique la détermination des 8 premiers moments. Le système linéaire équation (6) est réécrit en utilisant les moments «standard » de la variable aléatoire $X$ normée et centrée à la place des moments définis par l'équation (3) afin de simplifier les procédures de calculs numériques. Ces problèmes de précision numérique et le fait d'approximer la fonction de densité sur un intervalle $\left[b_{1}, b_{2}\right]$ conduisent à être très critique au regard de l'approximation des queues de distributions auxquelles correspondent des probabilités de défaillance très faibles.

\section{Éléments de vérification et de validation de la méthodologie proposée.}

Afin de vérifier la robustesse de la méthode proposée et de valider la pertinence des résultats qu'elle fournit, des procédures alternatives ont été mises en place. On suggère de comparer les résultats de la méthode proposée à ceux que fourniraient :

- des analyses FORM (First Order Reliability Method) [20] par « court-circuit » consistant à rassembler un ou plusieurs niveaux successifs de l'arbre de défaillance et à déterminer, point par point, la fonction de densité d'une variable aléatoire considérée;

- des simulations de Monte Carlo (MC) en ne réalisant des tirages que sur les variables de base $\left\{X^{(0)}\right\}$. Les niveaux intermédiaires sont également « court-circuités » et on obtient une caractérisation point par point de la densité de probabilité d'une variable aléatoire donnée.

\section{Analyses de fiabilité et de sensibilité au sein d'arbres de défaillance}

\subsection{Détermination directe de probabilités de défaillance et d'indices de fiabilité}

Un problème fondamental dans la théorie de la fiabilité des systèmes mécaniques consiste à évaluer l'intégrale multiple

$$
P_{\mathrm{f}}=\int_{g(\{x\}) \leq 0} f_{X_{1} \ldots X_{n v a}}(\{x\}) \mathrm{d} x_{1} \ldots \mathrm{d} x_{n v a}
$$

où $f_{X_{1} \ldots X_{n v a}}(\{x\})$ est la fonction de densité conjointe de $\{X\}=\left(X_{1}, \ldots, X_{n v a}\right)^{T}$, vecteur des paramètres 
aléatoires (chargements, propriétés matériaux, dimensions...) ; $g(\{x\})$ est la fonction d'état-limite ou de performance et $g(\{x\}) \leq 0$, le domaine d'intégration correspondant au domaine de défaillance; et enfin $P_{\mathrm{f}}$ représente la probabilité de défaillance $[5,21]$.

Dans notre cas, la fonction de performance est de la forme $G_{k}^{(l)}=M_{k}^{(l)}\left(\left\{X^{(l-1)}\right\}\right)$. Une approximation $\hat{f}_{G_{k}^{(l)}}(x)$ de la fonction de densité de probabilité de $G_{k}^{(l)}$ est donnée par l'équation (4) sous la forme

$$
\hat{f}_{G_{k}^{(l)}}(x)=c_{G_{k}^{(l)}}^{(n)} e^{Q_{G_{k}^{(l)}}^{(n)}(x)}
$$

avec

$$
Q_{G_{k}^{(l)}}^{(n)}(x)=\left\{\begin{array}{cc}
\sum_{i=1}^{n} a_{i} x^{i} & x \in\left[b_{1}, b_{2}\right] \\
-\infty & \text { sinon }
\end{array}(n \geq 2)\right.
$$

Ainsi, le problème (7) s'écrit plus simplement de la manière suivante

$$
\begin{aligned}
P_{\mathrm{f}}= & \int_{G_{k}^{(l)}\left(\left\{X^{(l-1)}\right\}\right) \leq 0} f_{X_{1}^{(l-1)} \ldots X_{n v a_{l-1}}^{(l-1)}} \\
& \times\left(\left\{x^{(l-1)}\right\}\right) \mathrm{d} x_{1}^{(l-1)} \ldots \mathrm{d} x_{n v a_{l-1}}^{(l-1)} \\
\approx & \int_{b_{1}}^{0} \hat{f}_{G_{k}^{(l)}}(v) \mathrm{d} v=\int_{b_{1}}^{0} c_{G_{k}^{(l)}}^{(n)} e^{Q_{G_{k}^{(l)}}^{(n)}(v)} \mathrm{d} v
\end{aligned}
$$

d'où on peut tirer une estimation au premier ordre de l'indice de fiabilité $\beta$ associé

$$
\beta \approx-\Phi^{-1}\left(\hat{P}_{\mathrm{f}}\right)
$$

dans laquelle $\Phi(x)=\frac{1}{\sqrt{2 \pi}} \int_{-\infty}^{x} \exp \left(-\frac{u^{2}}{2}\right) \mathrm{d} u$ est la fonction de répartition normale standard.

La détermination directe de la probabilité de défaillance et de l'indice de fiabilité peut être étendue au cas des variables de type $S_{k}^{(l)}$ dès lors qu'une fonction de type seuil $S_{k}^{(l)}-\lambda$ est déclarée. En effet, à partir de l'approximation $\hat{f}_{S_{k}^{(l)}}(x)$ de la fonction de densité de probabilité de $S_{k}^{(l)}$, il vient, au seuil d'étude $\lambda<b_{2}$

$$
\begin{aligned}
P_{\mathrm{f}}= & \int_{S_{k}^{(l)}\left(\left\{X^{(l-1)}\right\}\right) \leq \lambda} f_{X_{1}^{(l-1)} \ldots X_{n v a_{l-1}}^{(l-1)}} \\
& \times\left(\left\{x^{(l-1)}\right\}\right) \mathrm{d} x_{1}^{(l-1)} \ldots \mathrm{d} x_{n v a_{l-1}}^{(l-1)} \\
\approx & \int_{b_{1}}^{\lambda} \hat{f}_{S_{k}^{(l)}}(v) \mathrm{d} v=\int_{b_{1}}^{\lambda} c_{S_{k}^{(n)}}^{(n)} e^{Q_{S_{k}^{(l)}}^{(n)}(v)} \mathrm{d} v
\end{aligned}
$$

Les méthodes FORM et MC par « court-circuit » permettent aussi de vérifier et de valider les résultats obtenus sur les probabilités de défaillance.

\subsection{Analyse de sensibilité mécano-fiabiliste}

Connaître le poids d'une variable, c'est identifier l'influence de sa variation sur l'état du système mécanique.
Le but d'une étude de sensibilité mécano-fiabiliste est de sélectionner les variables les plus significatives, ce qui permet de mieux les maîtriser selon leur rôle par rapport au comportement mécanique ou à la fiabilité. L'importance d'une variable est considérée d'une part par rapport à la fonction de performance et d'autre part par rapport à la fiabilité.

La raison d'être des sensibilités mécano-fiabilistes réside dans le fait que les variables peuvent avoir une faible influence mécanique, mais présenter une grande dispersion probabiliste. Ainsi on peut imaginer la situation où une variable n'aurait qu'un petit effet sur la fonction de performance, mais dont la variabilité de la donnée pourrait jouer un rôle prépondérant dans la défaillance. Les analyses de sensibilité et d'importance déployées ici reposent sur l'exploitation des résultats fournis par les méthodes d'approximation au premier ordre de type FORM.

Typiquement, l'indice de fiabilité $\beta$ est lié au point $\left\{u^{*}\right\}$ (point de défaillance le plus probable exprimé dans l'espace des variables normales standard) par la relation

$$
\beta=-\{\alpha\}^{T}\left\{u^{*}\right\}
$$

où $\{\alpha\}=\frac{\left\{\nabla H\left(\left\{u^{*}\right\}\right)\right\}}{\left\|\left\{\nabla H\left(\left\{u^{*}\right\}\right)\right\}\right\|}$ est le vecteur des cosinus directeurs de la fonction d'état-limite $H$ écrite en fonction des variables normées. Les éléments du vecteur unitaire $\{\alpha\}$ indiquent la nature (sollicitation ou résistance) et l'importance des variables dans l'espace des variables normales standard. Cependant, les informations données par le vecteur $\{\alpha\}$ ne fournissent pas nécessairement d'indications sur la nature et l'importance des variables dans l'espace des variables physiques. Ainsi, il est nécessaire d'introduire le vecteur $\{\gamma\}$ caractérisant l'importance des variables dans l'espace d'origine. En considérant la transformation vers l'espace normal standard $\{u\}=T(\{x\})$ et son inverse $\{x\}=T^{-1}(\{u\})$, ainsi que le point de conception correspondant dans l'espace d'origine $\left\{x^{*}\right\}=$ $T^{-1}\left(\left\{u^{*}\right\}\right)$, la version linéarisée de la transformation au point de conception s'écrit

$$
\begin{aligned}
\{u\} & \cong\left\{u^{*}\right\}+\left[J_{\{u\},\{x\}}\right]_{*}\left(\{x\}-\left\{x^{*}\right\}\right) \\
& =\left\{u^{*}\right\}+\left[J_{\{u\},\{x\}}\right]_{*}\left(\{\hat{x}\}-\left\{x^{*}\right\}\right)
\end{aligned}
$$

où $\left[J_{\{u\},\{x\}}\right]_{*}$ est le Jacobien de la transformation $\{u\}=$ $u(\{x\})$ déterminé au point de conception et dans laquelle $\{\hat{x}\}$ est considéré comme un « équivalent normal $»$ de $\{x\}$ également au point de conception. On introduit alors le vecteur d'importance $\{\gamma\}[22]$ par la relation

$$
\{\gamma\}^{T}=\frac{\{\alpha\}^{T}\left[J_{\{u\},\{x\}}\right]_{*}[\hat{D}]}{\left\|\{\alpha\}^{T}\left[J_{\{u\},\{x\}}\right]_{*}[\hat{D}]\right\|}
$$

où $[\hat{D}]$ est la matrice diagonale des écarts-types de $\{\hat{x}\}$. Le rôle des distributions et des corrélations peut amplifier l'influence de certaines variables. Le résultat de l'étude d'importance est très utile dans le choix des variables aléatoires à prendre en compte. En effet, il permet 
dans un premier temps de distinguer les variables de type sollicitation de celles de type résistance par le signe de la composante d'importance. De plus, la détermination du vecteur $\{\gamma\}$ permet d'identifier les variables dont l'influence est amplifiée ou atténuée, lors de la transformation mécanique et de la transformation isoprobabiliste. Ainsi, nous pouvons limiter l'analyse mécano-fiabiliste aux variables les plus influentes et adapter la conception aux moyens de ces mêmes variables influentes.

Afin de mesurer l'impact potentiel de la variation d'une variable sur l'indice de fiabilité, on peut également considérer les sensibilités de $\beta$ aux variables aléatoires de bases $\{x\}$ calculées au point de conception $\left.\frac{\partial \beta}{\partial x_{i}}\right|_{\left\{x^{*}\right\}}$, ou bien les élasticités $e_{i}=\left.\frac{\partial \beta}{\partial x_{i}} \frac{x_{i}}{\beta}\right|_{\left\{x^{*}\right\}}$ qui sont adimensionnelles.

\section{Application à l'étude de la rupture d'un arbre de transmission}

\subsection{Description mécano-fiabiliste du problème}

Un arbre épaulé de boîte de vitesses est soumis à un chargement mécanique cyclique de flexion et de torsion et à un chargement statique de traction/compression. Les figures 4 et 5 représentent respectivement la schématisation par blocs de transfert de l'arbre de défaillance décrivant la rupture de l'arbre de boîte de vitesses et le modèle mécanique utilisé, la géométrie de l'arbre de boîte de vitesses étant volontairement simplifiée. Sous l'effet de ces sollicitations, un risque de rupture fragile brutale et un risque d'amorçage de fissuration par fatigue polycyclique sont redoutés. Cet exemple est adapté de [23]. L'ensemble des grandeurs physiques intervenant dans cette étude est répertorié dans les tableaux 1-3. On distingue les variables de base (Tab. 1) dont les hypothèses sur les distributions sont rappelées dans le tableau 2, des variables générées (Tab. 3) à travers des modèles mécaniques (Tab. 4) au fur et à mesure de la progression vers le sommet de l'arbre.

Le critère de plasticité de Von Mises [24] est utilisé pour caractériser le mode de défaillance « rupture ». Le critère de fiabilité associé à cet événement s'exprime à travers la fonction de performance $G_{1}$ définie par

$$
G_{1}=R_{m}-\sigma_{\mathrm{e}}^{\max }
$$

où $R_{m}$ est la limite à la rupture et $\sigma_{\mathrm{e}}^{\max }$ la contrainte équivalente de Von Mises maximum.

Le critère de fissuration par fatigue multi-axiale polycyclique à grand nombre de cycles de Dang Van [25,26], généralement utilisé dans le domaine de l'automobile caractérise quant à lui le second mode de défaillance considéré. Le principe de ce critère est de représenter les trajets de chargements en tout point d'une pièce mécanique dans un diagramme $[P(t)$ - pression hydrostatique, $\tau(t)$ - amplitude de cisaillement] limité par une droite dite de dommage (représentant la limite d'endurance intrinsèque du matériau) définie à partir d'essais simples uniaxiaux de traction, torsion ou flexion. Le



Fig. 4. Modélisation de l'arbre de boîte de vitesse.

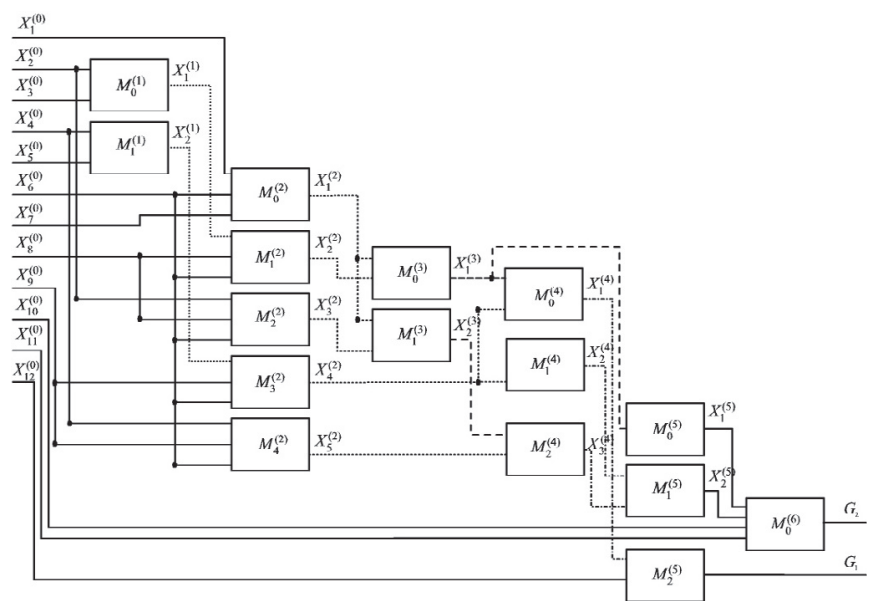

Fig. 5. Représentation par blocs de transfert de l'arbre de défaillance décrivant la rupture de l'arbre de boîte de vitesse.

critère de fiabilité associé à cet événement « fissuration par fatigue polycyclique $»$ s'exprime ainsi à travers la fonction de performance $G_{2}$ définie par

$$
G_{2}=\tau_{0}-[|\tau|-|\alpha| P]
$$

où $\tau_{0}$ et $\alpha$ sont les paramètres de la droite de Dang Van et correspondent aux caractéristiques de résistance du matériau en fatigue multi-axiale. Ces paramètres sont obtenus par des essais de fatigue de torsion et flexion uniaxiaux pour un nombre de cycles donné $\left(10^{6}, 10^{7}\right.$, ou $\left.10^{8}\right)$.

L'intérêt étant, ici, de montrer les produits de la méthode proposée, l'analyse complète du problème (i.e. la détermination du tenseur des contraintes macroscopiques, la détermination de l'amplitude de cisaillement et de la pression hydrostatique mésoscopique locale, la modélisation des critères de fiabilité) permettant l'identification des modèles mécaniques, tels qu'ils sont proposés dans le tableau 4, n'est pas rappelée [23,27].

\subsection{Résultats}

D'une part, les figures 6-9 permettent de visualiser respectivement les indices de fiabilité à un seuil $\lambda$ donné ainsi que les fonctions de répartition des variables aléatoires $G_{1}$ et $G_{2}$ obtenues par les méthodes EPM, FORM et MC. Les probabilités de défaillance et les indices de fiabilité obtenus avec les méthodes EPM, FORM 
Tableau 1. Définition des paramètres de base du système mécanique « arbre de boîte de vitesse ».

\begin{tabular}{llcc}
\hline Variable & \multicolumn{1}{c}{ Signification physique } & Modèle & Unité \\
\hline$F_{\text {tra }}$ & Effort de compression & $X_{1}^{(0)}$ & $\mathrm{N}$ \\
$M_{\mathrm{fle}}^{\text {moy }}$ & Moment de flexion moyen & $X_{2}^{(0)}$ & $\mathrm{N} . \mathrm{m}$ \\
$M_{\mathrm{fle}}^{\mathrm{a}}$ & Amplitude du moment de flexion & $X_{3}^{(0)}$ & $\mathrm{N} . \mathrm{m}$ \\
$M_{\mathrm{tor}}^{\mathrm{moy}}$ & Moment de torsion moyen & $X_{4}^{(0)}$ & $\mathrm{N} . \mathrm{m}$ \\
$M_{\mathrm{tor}}^{\mathrm{a}}$ & Amplitude du moment de torsion & $X_{5}^{(0)}$ & $\mathrm{N} . \mathrm{m}$ \\
$d$ & Diamètre de l'arbre de boîte de vitesses & $X_{6}^{(0)}$ & $\mathrm{m}$ \\
$K_{\mathrm{t}}^{\mathrm{fle}}$ & Coefficient de concentration de contrainte de flexion & $X_{7}^{(0)}$ & - \\
$K_{\mathrm{t}}^{\text {tra }}$ & Coefficient de concentration de contrainte de compression & $X_{8}^{(0)}$ & - \\
$K_{\mathrm{t}}^{\text {tor }}$ & Coefficient de concentration de contrainte de cisaillement & $X_{9}^{(0)}$ & - \\
$\tau_{0}$ & Paramètre de la droite de Dang Van & $X_{10}^{(0)}$ & $\mathrm{Pa}$ \\
$\alpha$ & Paramètre de la droite de Dang Van & $X_{11}^{(0)}$ & - \\
$R_{m}$ & Limite à la rupture & $X_{12}^{(0)}$ & $\mathrm{Pa}$ \\
\hline
\end{tabular}

Tableau 2. Caractérisation stochastique des paramètres de base.

\begin{tabular}{ccccc}
\hline Variable & Loi de distribution & Moyenne & Écart-type & Coefficient de variation \\
\hline$F_{\text {tra }}$ & Loi normale & $8,968 \times 10^{4} N$ & $1,7938 \times 10^{4} \mathrm{~N}$ & 0,2 \\
$M_{\mathrm{fle}_{\text {moy }}}$ & Loi normale & $4,349 \times 10^{4} \mathrm{~N} . \mathrm{mm}$ & $4,349 \times 10^{3} \mathrm{~N} . \mathrm{mm}$ & 0,1 \\
$M_{\text {fle }}$ & Loi normale & $8,697 \times 10^{5} \mathrm{~N} . \mathrm{mm}$ & $2,6091 \times 10^{5} \mathrm{~N} . \mathrm{mm}$ & 0,3 \\
$M_{\text {tor }_{\text {moy }}}$ & Loi normale & $2,451 \times 10^{6} \mathrm{~N} \cdot \mathrm{mm}$ & $2,451 \times 10^{5} \mathrm{~N} . \mathrm{mm}$ & 0,1 \\
$M_{\text {tor }}$ & Loi normale & $1,471 \times 10^{6} \mathrm{~N} \cdot \mathrm{mm}$ & $2,942 \times 10^{5} \mathrm{~N} . \mathrm{mm}$ & 0,2 \\
$d$ & Loi normale & $65 \mathrm{~mm}$ & $0,975 \mathrm{~mm}$ & 0,015 \\
$K_{\mathrm{t}}^{\text {tra }}$ & Loi lognormale & 3,7 & 0,37 & 0,1 \\
$K_{\mathrm{t}}^{\text {fle }}$ & Loi lognormale & 3,1 & 0,31 & 0,1 \\
$K_{\mathrm{t}}^{\text {tor }}$ & Loi lognormale & 2,2 & 0,22 & 0,1 \\
$\tau_{0}$ & Loi normale & $310 \mathrm{MPa}$ & $46,5 \mathrm{MPa}$ & 0,15 \\
$\alpha$ & Loi lognormale & $\pm 0,36$ & 0,054 & $\pm 0,15$ \\
$R_{m}$ & Loi normale & $1200 \mathrm{MPa}$ & $180 \mathrm{MPa}$ & 0,15 \\
\hline
\end{tabular}

Tableau 3. Signification physique des paramètres intermédiaires de l'étude de la rupture de l'arbre de boîte de vitesse.

\begin{tabular}{cclc}
\hline Modèle & Variable associée & Signification physique & Unité \\
\hline$X_{1}^{(1)}$ & $M_{\mathrm{fe}}^{\max }$ & Moment de flexion maximal & $\mathrm{N} . \mathrm{m}$ \\
$X_{2}^{(1)}$ & $M_{\mathrm{tor}}^{\max }$ & Moment de torsion maximal & $\mathrm{N} . \mathrm{m}$ \\
$X_{1}^{(2)}$ & $\sigma_{\mathrm{tra}}$ & Contrainte macroscopique de compression & $\mathrm{Pa}$ \\
$X_{2}^{(2)}$ & $\sigma_{\mathrm{fle}}^{\max }$ & Contrainte macroscopique de flexion maximale & $\mathrm{Pa}$ \\
$X_{3}^{(2)}$ & $\sigma_{\mathrm{fle}}^{\operatorname{moy}}$ & Contrainte macroscopique de flexion moyenne & $\mathrm{Pa}$ \\
$X_{4}^{(2)}$ & $\tau_{\mathrm{tor}}^{\max }$ & Contrainte macroscopique de cisaillement maximum & $\mathrm{Pa}$ \\
$X_{5}^{(2)}$ & $\tau_{\mathrm{tor}}^{\operatorname{moy}}$ & Contrainte macroscopique de cisaillement moyenne & $\mathrm{Pa}$ \\
$X_{1}^{(3)}$ & $\sigma^{\max }$ & Contrainte de traction-compression maximum & $\mathrm{Pa}$ \\
$X_{2}^{(3)}$ & $\sigma^{\operatorname{moy}}$ & Contrainte de traction-compression moyenne & $\mathrm{Pa}$ \\
$X_{1}^{(4)}$ & $\sigma_{\mathrm{e}}^{\max }$ & Contrainte équivalente de Von Mises maximum & $\mathrm{Pa}$ \\
$X_{2}^{(4)}$ & $T_{\max }$ & Cisaillement mésoscopique local maximum & $\mathrm{Pa}$ \\
$X_{3}^{(4)}$ & $T_{0}$ & Cisaillement mésoscopique local moyen & $\mathrm{Pa}$ \\
$G_{1}$ & $G_{1}$ & « Rupture & $\mathrm{Pa}$ \\
$X_{1}^{(5)}$ & $\tau$ & Amplitude de cisaillement mésoscopique local & $\mathrm{Pa}$ \\
$X_{2}^{(5)}$ & $p$ & Pression mésoscopique locale & $\mathrm{Pa}$ \\
$G_{2}$ & $G_{2}$ & « Fissuration par fatigue & $\mathrm{Pa}$ \\
\hline
\end{tabular}


Tableau 4. Modèles mécaniques intervenant dans la description de la rupture de l'arbre de boîte de vitesse.

\begin{tabular}{|c|c|c|}
\hline Niveau & Modèle mécanique & Transformation associée \\
\hline 1 & $\begin{array}{l}M_{0}^{(1)} \longmapsto X_{1}^{(1)} \\
M_{1}^{(1)} \longmapsto X_{2}^{(1)}\end{array}$ & $\begin{array}{l}M_{\mathrm{fl}}^{\max }=M_{\mathrm{fle}}^{\mathrm{moy}}+M_{\mathrm{fle}}^{\mathrm{a}} \\
M_{\mathrm{tor}}^{\max }=M_{\mathrm{tor}}^{\mathrm{moy}}+M_{\mathrm{tor}}^{\mathrm{a}}\end{array}$ \\
\hline 2 & $\begin{aligned} & M_{0}^{(2)} \longmapsto X_{1}^{(2)} \\
& M_{1}^{(2)} \longmapsto X_{2}^{(2)} \\
& M_{2}^{(2)} \longmapsto X_{3}^{(2)} \\
& M_{3}^{(2)} \longmapsto X_{4}^{(2)} \\
& M_{4}^{(2)} \longmapsto X_{5}^{(2)}\end{aligned}$ & $\begin{array}{c}\sigma_{\mathrm{tra}}=K_{\mathrm{t}}^{\mathrm{tra}} \frac{4 F}{\pi d^{2}} \\
\sigma_{\mathrm{fle}}^{\max }=K_{\mathrm{t}}^{\mathrm{fle}} \frac{32 M_{\mathrm{fle}}}{\pi d^{3}} \\
\sigma_{\mathrm{fle}}^{\operatorname{moy}}=K_{\mathrm{t}}^{\mathrm{fle}} \frac{32 M_{\mathrm{fle}}}{\pi d^{3}} \\
\tau_{\mathrm{tor}}^{\max }=K_{\mathrm{t}}^{\mathrm{tor}} \frac{16 M_{\mathrm{tor}}}{\pi d^{3}} \\
\tau_{\text {tor }}^{\operatorname{moy}}=K_{\mathrm{t}}^{\text {tor }} \frac{16 M_{\mathrm{tor}}^{\mathrm{moy}}}{\pi d^{3}}\end{array}$ \\
\hline 3 & $\begin{array}{l}M_{0}^{(3)} \longmapsto X_{1}^{(3)} \\
M_{1}^{(3)} \longmapsto X_{2}^{(3)} \\
\end{array}$ & $\begin{aligned} \sigma^{\mathrm{max}} & =\sigma_{\mathrm{fle}}^{\max }+\sigma_{\text {tra }} \\
\sigma^{\mathrm{moy}} & =\sigma_{\mathrm{fle}}^{\mathrm{moy}}+\sigma_{\text {tra }}\end{aligned}$ \\
\hline 4 & $\begin{aligned} & M_{0}^{(4)} \longmapsto X_{1}^{(4)} \\
& M_{1}^{(4)} \longmapsto X_{2}^{(4)} \\
& M_{2}^{(4)} \longmapsto X_{3}^{(4)}\end{aligned}$ & $\begin{aligned} \sigma_{\mathrm{e}}^{\max } & =\sqrt{\left(\sigma^{\max }\right)^{2}+3\left(\tau_{\text {tor }}^{\max }\right)^{2}} \\
T_{\max } & =\sqrt{\left(\frac{\sigma^{\max }}{2}\right)^{2}+\left(\tau_{\text {tor }}^{\max }\right)^{2}} \\
T_{0} & =\sqrt{\left(\frac{\sigma_{\text {moy }}}{2}\right)^{2}+\left(\tau_{\text {tor }}^{\text {moy }}\right)^{2}}\end{aligned}$ \\
\hline 5 & $\begin{aligned} M_{0}^{(5)} & \longmapsto G_{1} \\
M_{1}^{(5)} & \longmapsto X_{1}^{(5)} \\
M_{2}^{(5)} & \longmapsto X_{2}^{(5)}\end{aligned}$ & $\begin{array}{l}G_{1}=R_{m}-\sigma_{\mathrm{e}} \\
\tau=T^{\max }-T_{0} \\
\quad p=\frac{1}{3} \sigma^{\max }\end{array}$ \\
\hline 6 & $M_{0}^{(6)} \longmapsto G_{2}$ & $G_{2}=\tau_{0}-[|\tau|+|\alpha| p]$ \\
\hline
\end{tabular}

et $\mathrm{MC}$, pour les fonctions de performances $G_{1}=0$ et $G_{2}=0$, sont présentés dans le tableau 5. La comparaison des résultats fournis par les trois méthodes montre que l'approximation par EPM est satisfaisante et peut être utilisée comme alternative aux méthodes FORM et MC. L'EPM permet d'obtenir une approximation continue de la fonction de densité d'une variable aléatoire alors que les méthodes FORM et MC ne fournissent qu'une reconstruction point par point de cette densité. Sans être plus rapide que la méthode FORM appliquée à la reconstruction fine de la densité, l'EPM est en ce sens plus avantageuse que la simulation de Monte Carlo.

D'autre part, les élasticités de $\beta$ par rapport aux variables aléatoires de base ainsi que les facteurs d'importance de $G_{1}$ et $G_{2}$ par rapport à ces mêmes variables sont répertoriés dans les tableaux 6 et 7 .

Enfin, les résultats caractéristiques que fournit l'approche proposée, à savoir les chaînes des facteurs d'importance de l'arbre caractérisant la défaillance du composant mécanique, sont illustrés par les tableaux 8 et 9 .

En pratique, les risques de rupture ainsi que les risques de fissuration par fatigue polycyclique sont pris en compte dès la conception d'un composant mécanique de groupe motopropulseur. Ici, la nature simplifiée du problème et les hypothèses réalisées sur les dispersions des variables ne permettent pas de conclure sur les valeurs observées au niveau des indices de fiabilité associés aux fonctions de performances $G_{1}$ et $G_{2}$ (Tab. 5). En revanche, il est possible de conclure sur l'importance relative de chacun des paramètres de base et de leurs dispersions dans la perte de fonction de l'arbre épaulé. L'analyse fiabiliste et l'analyse de sensibilité mises en œuvre permettent de caractériser leur impact sur les deux modes de défaillance identifiés précédemment.



Fig. 6. Indice de fiabilité $\beta$ associé à la fonction $G_{1}$ en fonction du seuil d'étude $\lambda$.

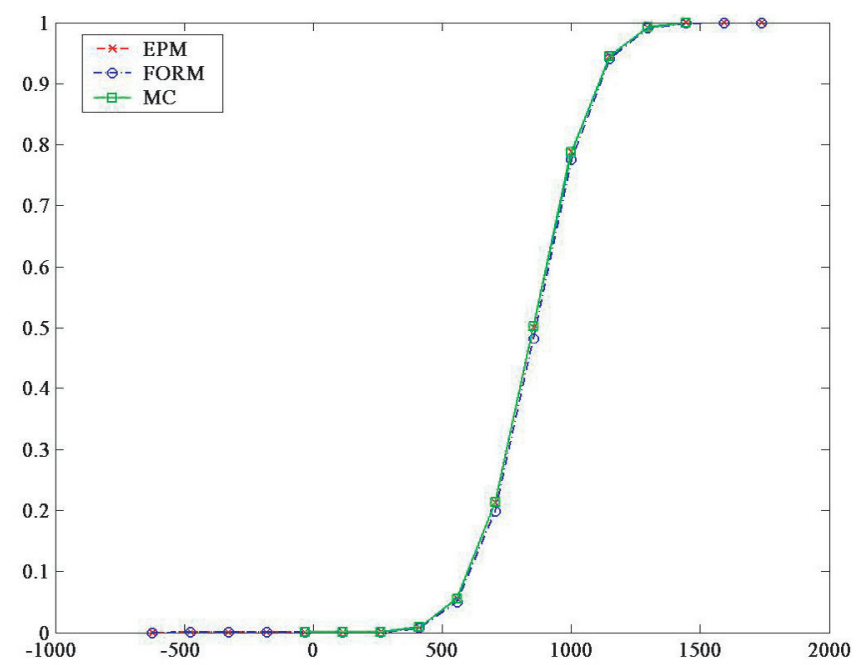

Fig. 7. Fonction de répartition de la variable aléatoire $G_{1}$ obtenue par les méthodes EPM, FORM et MC.

\subsubsection{Mode de défaillance : rupture}

Ce mode de défaillance est régi par la fonction $G_{1}$. L'étude des signes des composantes du vecteur d'importance $\{\gamma\}$ nous renseigne sur le caractère résistant ou contraignant des paramètres ainsi que sur les variations de $G_{1}$ au voisinage du point de conception (Tab. 6). Du point de vue de l'indice de fiabilité, la deuxième colonne du tableau 6 , montre que les paramètres principaux influant sur la valeur de l'indice de fiabilité de l'arbre épaulé par rapport à une éventuelle rupture sont le diamètre de l'arbre $d$, la limite à la rupture $R_{m}$, le coefficient de concentration de contrainte $K_{\mathrm{t}}^{\mathrm{tor}}$, le moment de torsion $M_{\text {tor }}^{\text {moy }}$ et son amplitude $M_{\mathrm{tor}}^{\mathrm{a}}$. Il en résulte qu'au moment de la conception un intérêt très particulier doit être porté à l'étude, à la définition voire à l'optimisation des paramètres $d, R_{m}$, $K_{\mathrm{t}}^{\mathrm{tor}}, M_{\mathrm{tor}}^{\text {moy }}$ et $M_{\mathrm{tor}}^{\mathrm{a}}$ dans l'objectif de satisfaire un niveau de fiabilité exigé. 
Tableau 5. Probabilités de défaillance et indices de fiabilité EPM, FORM et MC associés aux événements $G_{1}$ et $G_{2}$.

\begin{tabular}{ccccccc}
\hline $\begin{array}{c}\text { Scénario de } \\
\text { défaillance }\end{array}$ & \multicolumn{2}{c}{ Probabilité de défaillance $P_{\mathrm{f}}$} & \multicolumn{3}{c}{ Indice de fiabilité $\beta$} \\
$G_{1}$ & $1,65 \times 10^{-6}$ & $2,12 \times 10^{-6}$ & $2,15 \times 10^{-6}$ & FORM & EPM & MC \\
\hline$G_{2}$ & $1,62 \times 10^{-5}$ & $8,41 \times 10^{-5}$ & $2,95 \times 10^{-5}$ & 4,16 & 4,60 & 4,60 \\
& & & & & & \\
\hline
\end{tabular}

Tableau 6. Rupture, fonction de performance $G_{1}$ : élasticités de $\beta$ et vecteur d'importance $\{\alpha\}$.

\begin{tabular}{ccccc}
\hline $\begin{array}{c}\text { Variables } \\
\text { de base }\end{array}$ & $\begin{array}{c}\text { Elasticités de } \beta \text { aux } \\
\text { v.a.physiques }\left.\frac{\partial \beta}{\partial x_{i}} \frac{x_{i}}{\beta}\right|_{\left\{x^{*}\right\}}\end{array}$ & \multicolumn{2}{c}{ Vecteur d'importance } & Caractère \\
\hline$R_{m}$ & $-0,4501$ & 0,9683 & 93,76 & Résistance \\
$K_{\mathrm{t}}^{\text {fle }}$ & 0,08074 & $-0,03747$ & 0,14 & Sollicitation \\
$M_{\mathrm{fle}}^{\text {moy }}$ & $3,495 \times 10^{-3}$ & $-1,624 \times 10^{-3}$ & 0,00 & Sollicitation \\
$M_{\mathrm{fle}}^{\mathrm{a}}$ & 0,07725 & $-0,1055$ & 1,10 & Sollicitation \\
$d$ & $-1,2768$ & 0,08965 & 0,80 & Résistance \\
$K_{\mathrm{t}}^{\text {tra }}$ & 0,07344 & $-0,03408$ & 0,12 & Sollicitation \\
$F$ & 0,07344 & $-0,06446$ & 0,42 & Sollicitation \\
$K_{\mathrm{t}}^{\text {tor }}$ & 0,2959 & $-0,13731$ & 1,89 & Sollicitation \\
$M_{\mathrm{tor}}^{\text {moy }}$ & 0,1822 & $-0,08164$ & 0,67 & Sollicitation \\
$M_{\text {tor }}^{\mathrm{a}}$ & 0,1138 & $-0,1048$ & 1,10 & Sollicitation \\
\hline
\end{tabular}



Fig. 8. Indice de fiabilité $\beta$ associé à la fonction $G_{2}$ en fonction du seuil d'étude $\lambda$.

L'étude d'importance (Tabs. 6 et 8) exhibe, quant à elle, la contribution prédominante de $R_{m}$ à la fonction de performance $G_{1}$ ainsi que l'influence de la contrainte de cisaillement maximale $\tau_{\text {tor }}^{\max }$ sur la valeur de la contrainte équivalente maximale $\sigma_{\mathrm{e}}^{\max }$. Toutefois, ce type de scénario de défaillance ne permet pas d'utiliser au mieux la méthode proposée dont l'intérêt pratique est mis en avant par l'étude du second mode de défaillance.

\subsubsection{Mode de défaillance : amorçage de fissuration par fatigue}

Ce mode de défaillance est régi par la fonction $G_{2}$. L'étude des signes des composantes du vecteur

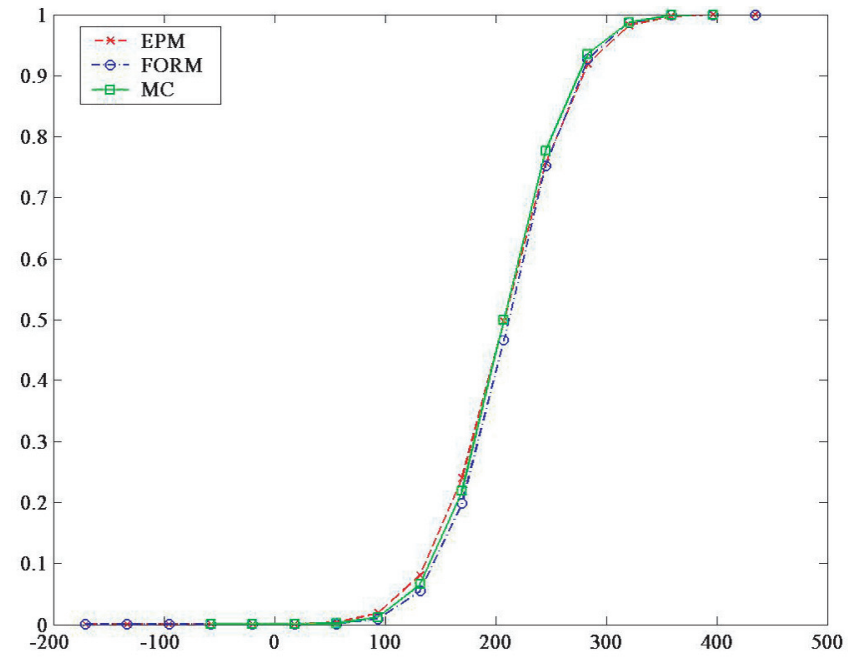

Fig. 9. Fonction de répartition de la variable aléatoire $G_{2}$ obtenue par les méthodes EPM, FORM et MC.

d'importance $\{\gamma\}$ renseigne sur le caractère résistant ou contraignant des paramètres ainsi que sur les variations de $G_{2}$ au voisinage du point de conception. L'intérêt d'une telle étude est ici bien illustré par le comportement de $G_{2}$ par rapport au moment de torsion moyen $M_{\text {tor }}^{\text {moy }}$. En effet, on constate d'après le tableau 7 , que la fonction de performance $G_{2}$ est croissante dans le sens des valeurs croissantes de $M_{\text {tor }}^{\text {moy }}$. La fiabilité du composant est donc augmentée vis-à-vis du risque de fissuration si la valeur nominale de $M_{\text {tor }}^{\text {moy }}$ est augmentée. Ceci peut paraître surprenant mais s'explique par le fait que le $M_{\mathrm{tor}}^{\text {moy }}$ contribue fortement à l'augmentation de $T_{0}$ par le biais de $\tau_{\text {tor }}^{\text {moy }}$ (Tabs. 7 et 9 ). Du point de vue de l'indice de fiabilité, la 
Tableau 7. Amorçage de fissuration par fatigue, fonction de performance $G_{2}$ : élasticités de $\beta$ et vecteur d'importance $\{\alpha\}$.

\begin{tabular}{ccccc}
\hline Variables & Elasticités de $\beta$ aux & \multicolumn{2}{c}{ Vecteur d'importance } & Caractère \\
de base & v.a.physiques $\left.\frac{\partial \beta}{\partial x_{i}} \frac{x_{i}}{\beta}\right|_{\left\{x^{*}\right\}}$ & $\{\gamma\}$ & $\%$ & \\
\hline$\tau_{0}$ & $-0,6396$ & 0,8616 & 74,23 & Résistance \\
$K_{\mathrm{t}}^{\text {fle }}$ & 0,3278 & $-0,1359$ & 1,84 & Sollicitation \\
$M_{\mathrm{fle}}^{\text {moy }}$ & $4,691 \times 10^{-3}$ & $-1,948 \times 10^{-3}$ & 0,00 & Sollicitation \\
$M_{\mathrm{fle}}^{\mathrm{a}}$ & 0,3231 & $-0,3942$ & 15,53 & Sollicitation \\
$d$ & $-1,8238$ & 0,1145 & 1,30 & Résistance \\
$K_{\mathrm{t}}^{\text {tra }}$ & 0,09493 & $-0,03936$ & 0,16 & Sollicitation \\
$F$ & 0,09493 & $-0,07433$ & 0,54 & Sollicitation \\
$K_{\mathrm{t}}^{\text {tor }}$ & 0,2169 & $-0,08992$ & 0,81 & Sollicitation \\
$M_{\mathrm{tor}}^{\text {moy }}$ & $-0,04275$ & 0,01788 & 0,03 & Résistance \\
$M_{\text {tor }}^{\mathrm{a}}$ & 0,2596 & $-0,2137$ & 4,56 & Sollicitation \\
$\alpha$ & 0,1579 & $-0,09791$ & 1,00 & Sollicitation \\
\hline
\end{tabular}

Tableau 8. Décomposition de l'arbre d'influence de la variable $G_{1}$.

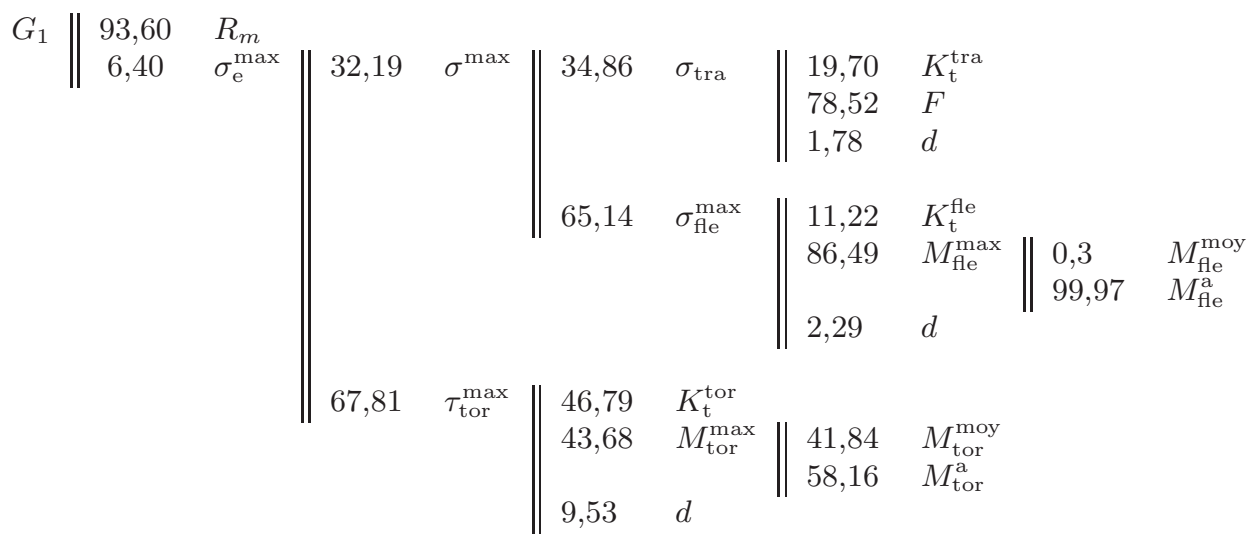

Tableau 9. Décomposition de l'arbre d'influence de la variable $G_{2}$.



deuxième colonne du tableau 7 montre que les paramètres principaux influant sur la valeur de l'indice de fiabilité de l'arbre épaulé par rapport à une éventuelle fissuration par fatigue sont le diamètre de l'arbre $d$ et la limite d'endurance en torsion alternée $\tau_{0}$. Ensuite, on constate que les coefficients de concentration de contrainte $K_{\mathrm{t}}^{\mathrm{fle}}$ et $K_{\mathrm{t}}^{\mathrm{tor}}$ ainsi que les amplitudes des moments de flexion $M_{\text {fle }}^{\mathrm{a}}$ et de torsion $M_{\text {tor }}^{\mathrm{a}}$ agissent de manière similaire sur la valeur de l'indice de fiabilité.
L'étude d'importance sur ce mode de défaillance $G_{2}$, qui est résumée dans le tableau 9, montre que deux paramètres $\tau$ et $\tau_{0}$ gouvernent majoritairement la défaillance. De plus, elle illustre très clairement l'apport pratique de la méthode proposée qui favorise l'identification des paramètres influents par la caractérisation des paramètres élémentaires de défaillance et la décomposition de la physique de défaillance. Pour cela il suffit de regarder, par exemple, la chaîne 
d'importance liée à la variable $\tau$, amplitude de cisaillement mésoscopique local.

\subsection{Conclusion}

L'application de la méthodologie proposée à l'étude de la rupture d'un arbre de boîte de vitesses démontre son intérêt multiple. En effet, elle permet d'abord de déterminer l'indice de fiabilité d'une configuration donnée. Dès la phase de conception, elle renseigne également l'ingénieur sur les phénomènes physiques et sur les paramètres stratégiques gouvernant un mécanisme de défaillance et ce à différents niveaux d'un arbre. En outre, elle informe sur la manière dont il doit les manipuler pour atteindre les objectifs de fiabilité qui lui sont imposés.

\section{Conclusions et perspectives}

L'exploitation quantitative selon une approche mécano-probabiliste de la modélisation par arbres de défaillance physiques de la fiabilité d'un système mécanique est réalisée en mettant en place une méthodologie de résolution complète, efficace et évolutive. L'objectif est de conduire des analyses de fiabilité en s'éloignant le moins possible de la physique associée aux mécanismes défaillants. Basée sur la technique d'approximation de fonctions de densité de probabilité de Er dite par « exponentielle d'un polynôme » et sur les techniques d'analyses de sensibilités et d'importance au premier ordre, cette méthode présente l'avantage de fournir des informations essentielles, en particulier au niveau de la caractérisation des phénomènes physiques mis en jeu et de la mesure de leur importance. La vérification et la validation des résultats obtenus sur l'exemple particulier de la rupture d'un arbre de boîte de vitesses par rapport aux méthodes FORM et aux techniques de simulation de Monte Carlo témoignent de l'intérêt pratique de la méthode proposée. La mise en œuvre de cette méthode et l'analyse de son comportement sur des exemples plus complexes, faisant par exemple intervenir des modèles mécaniques appelant un code éléments finis sont d'ores et déjà à l'étude.

Cette approche, dont des applications immédiates sont envisageables dans les domaines d'aide à la conception ou de validation de la conception, s'inscrit parfaitement dans une démarche d'ingénierie robuste. En effet, elle participe à la conception fiable et durable tout en favorisant le respect des impératifs de qualité, de coûts et de délais inhérents aux problèmes industriels. L'échange permanent et indispensable entre les différents acteurs impliqués dans ce type d'étude de fiabilité mécanique que sont le mécanicien, le fiabiliste, le probabiliste, le statisticien et le décideur assure l'efficacité et la réussite de la mise en œuvre d'une telle méthodologie.

\section{Références}

[1] A. Villemeur, Reliability, Availibility, Maintainability and Safety Analysis, Wiley \& Sons, New York, 1992

[2] P.D.T. O'Connor, D. Newton, R. Bromley, Practical Reliability Engineering, Fourth Edition, Wiley \& Sons, New York, 2002

[3] M. Modarres, M. Kaminskiy and Vasiliy Krivtsov, Reliability Engineering and Risk Analysis, A Practical Guide, Quality and Reliability, Marcel Dekker, Inc., 1999, p. 55

[4] E. Castillo, J.M. Sarabia, C. Solares, P. Gomez, Uncertainty analyses in fault trees and Bayesian networks using FORM/SORM methods, Reliability Engineering and System Safety 65 (1999) 29-40

[5] M. Lemaire, Fiabilité des Structures - Couplage MécanoFiabiliste Statique, Hermes Science Publication, 2005. En collaboration avec A. Chateauneuf et J.C. Mitteau, ISBN 2-7462-1057-6

[6] G-K. Er, A method for multi-parameter PDF estimation of random variables, Structural Safety 20 (1998) 341-355

[7] M. Pendola, Fiabilité des structures en contexte d'incertitudes statistiques et d'écarts de modélisation, Thèse, LaRAMA/IFMA, Université Blaise Pascal et Département Mécanique et Technologie des Composants, Électricité de France, Recherche \& Développement, 2000

[8] N.L. Johnson, Systems of frequency curves generated by methods of translation, Biometrika 36 (1949) 149-176

[9] E.S. Pearson, M. Tukey, Distributions whose first moments are known, Biometrika 6 (1965) 127-137.

[10] E.S. Pearson, H.O. Hartley, Biometrika tables for statisticians, Cambridge University Press, 1972

[11] H. Baldeweck, Méthodes des éléments finis stochastiques, Applications à la géotechnique et à la mécanique de la rupture, thèse, Université d'Évry Val d'Essonne, 1998

[12] A. Stuart, J.K. Ord, Kendall's advanced theory of statistics, Wiley \& Sons, New York, 1983

[13] S.R. Winterstein, Moment-Based Hermite Models of Random Vibration. Technical Report $\mathrm{n}^{\circ} 219$, Dept. of Structural Engineering, Technical University of Denmark, Lyngby, 1987

[14] Z. Zong, K.Y. Lam, Estimation of complicated distributions using B-spline functions, Structural Safety 20 (1998) 341-355

[15] Z. Zong, K.Y. Lam, Bayesian estimation of complicated distributions, Structural Safety 22 (2000) 81-95

[16] B. Puig, Modélisation et simulation de processus stochastiques non gaussiens, thèse de l'Université Pierre et Marie Curie, Paris VI, spécialité mathématiques, 2003

[17] B. Sudret, M. Berveiller, M. Lemaire, Eléments finis stochastiques spectraux, nouvelles perspectives, in Proc. $16^{\mathrm{e}}$ Congrès Français de Mécanique, Nice, 2003

[18] B. Sudret, I. Cherradi, Quadrature method for finite element reliability analysis, in Der Kiureghian, Madanat \& Pestana (Eds.), Proceedings of the 9th ICASP, Millpress, Rotterdam, 2003 
[19] J.N. Reddy, Applied functional analysis and variational methods in engineering, McGraw-Hill, New York, 1986

[20] N. Gayton, J.-P. Lambelin, M. Lemaire, Approche probabiliste du facteur de réduction de charge d'une coque mince, Mécanique \& Industries 3 (2002) 227-236

[21] O. Ditlevsen, H.O. Madsen, Structural reliability methods, Wiley \& Sons, New York, 1996

[22] A. Der Kurieghian, Lecture : CE229 Structural Reliability, University of California at Berkeley, 2002

[23] T. Moro, Analyse de sensibilité de la réponse mécanofiabiliste d'un composant GMP (Importance qualitative et quantitative des caractéristiques critiques), Rapport Technique Ligeron S.A Réf. 02-009-C/R01/TM, à l'attention de Renault s.a.s., 2003
[24] J. Lemaître, J.L. Chaboche, Mécanique des matériaux solides, Dunod, Paris, 1988

[25] K. Dang Van, G. Cailletaud, J.F. Flavenot, A. Le Douaron et H.P. Lieurade, Critère d'amorçage en fatigue à grands nombres de cycles sous sollicitations multiaxiales, Journées de Printemps de la SFM Paris, 1984, pp. 301-337

[26] P. Schimmerling, Fiabilité prévisionnelle en fatigue, Application du critère de Dang Van, Mécanique Matériaux Électricité 429 (janvier/mars 1989)

[27] A. Hähnel, Sûreté de fonctionnement de système mécanique de l'automobile, Mémoire de diplôme d'ingénieur, Institut Français de Mécanique Avancée, 2003 